\title{
Energy of Spinning Black Holes in XRBs and AGN
}

Dipo Mahto ${ }^{1 *}$, Md Shams Nadeem ${ }^{2}$, Umakant Prasad ${ }^{3}$ \& Kumari Vineeta ${ }^{4}$

${ }^{1}$ Assistant Professor, Dept. of Physics, Marwari College,T.M.B.U.Bhagalpur-812007, India

${ }^{2}$ Research Scholar, University Dept. of Physics, T. M. B. U. Bhagalpur- 812007, India

${ }^{3}$ Assistant Professor, Dept. of Physics, T.N.B. College, T. M. B. U. Bhagalpur-812007,India

${ }^{4}$ Lecturer in Physics, Dept. of Education, S.M. College,T.M.B.U.Bhagalpur-812007, India

\begin{abstract}
Aims: To justify the model for energy of spinning black holes $\left(E_{B H s}=K_{B H s} R_{s}^{\prime}\right)$ where $K_{B H s}$ is the black hole constant having the value $1.214 \times 10^{44} \mathrm{Jm}^{-1}$ as proposed Dipo Mahto et al. (2011).

Study Design: Data for the mass of black holes have collected from the research paper entitled :Super massive Black Holes in Galactic Nuclei: Past Present and Future Research(2005), Space Science Reviews by L. Ferrarese and H. Ford and Black holes in Astrophysics (2005), New Journal Physics by R. Narayan. The data for the energy of black holes are taken from the paper entitled: The nature of the energy source in radio galaxies and active galactic nuclei, International Astronomical Union (1982) by V. Pacini and M. Salvati and acceleration and radiation processes around active galactic nuclei, Astrophysics and Space Science (1985) by V. Krishan.
\end{abstract}

Place and Duration of Study: Department of Physics, Marwari College Bhagalpur and University Department of Physics, T.M.B.U. Bhagalpur, between October 2013 and February 2014.

Methodology: A theoretical based work using Laptop to calculate the calculation for energy of spinning black holes at Marwari College Bhagalpur and the residential research chamber of the first author.

Results: The calculation shows that the total energy of the rest masses for stellar-mass black holes $(M \sim 5-20$ $\left.M_{\circ}\right)$ in X-ray binaries is $f e w \times 10^{55}$ ergs and for the super massive black holes $\left(M \sim 10^{6}-10^{9.5} M_{o}\right)$ in active galactic nuclei is $f e w \times 10^{60}-10^{64} \mathrm{ergs}$.

Our result agrees with the result of other research work done previously by Pacini and Salvati and by Krishan and justifies the validity of this model.

Conclusion: The validity of model $\left(E_{B H s}=K_{B H S} R_{s}^{\prime}\right)$ is justified which agrees with the result of other research work done earlier by Pacini and Salvati (1982) and Krishan (1985).

Keywords: Event horizon, XRBs, AGN and Energy

\section{Introduction}

James Bardeen, Jacob Bekenstein, Carter, and Hawking have vital role to lead the formulation of the laws of black hole mechanics. The laws of black hole mechanics describe the behaviour of a black hole in close analogy to the laws thermodynamics by relating mass to energy, area to entropy, and surface gravity to temperature [1-3]. In October 23, 2001, scientists for the first time have seen energy being extracted from a black hole. Like an electric dynamo, this black hole spins and pumps energy out through cable-like magnetic field lines into the chaotic gas whipping around it, making the gas already internally hot from the sheer force of crushing gravity even hotter [4]. In 2011, Dipo Mahto et al. derived also an expression for the energy of spinning black holes in terms of the radius of event horizon given by the equation $E_{B H s}=K_{B H s} R_{s}^{\prime}$ where $K_{B H s}$ is the black hole constant having the value $1.214 \times 10^{44} \mathrm{~J} / \mathrm{m}$ for spinning black holes [5]. In the same year, Dipo Mahto et al. derived an expression for the change in energy and entropy of non-spinning black holes taking account the first law of the black hole mechanics relating the change in mass $\mathrm{M}$, angular momentum $\mathrm{J}$, horizon area $\mathrm{A}$ and charge Q, of a stationary black hole with Einstein's mass-energy equivalence relation [3]. In 2013, Dipo Mahto et al. justified the model for energy of non-spinning black holes $E_{B H}=K_{B H} R_{s}$ as proposed by Kanak Kumari et al. in 2010.

In the present paper, we have calculated the energy of different spinning black holes existing in XRBs and AGN on the basis of this model. The calculation shows that the total energy of the rest masses $M$ $\sim 5-20 \mathrm{M}_{\circ}$ of stellar - mass black holes in X-ray binaries is $f e w \times 10^{55}$ ergs and for the masses $\mathrm{M} \sim 10^{6}-10^{9.5} \mathrm{M}_{\mathrm{o}}$ of super massive black holes in active galactic nuclei is $f e w \times 10^{60}-10^{64}$ ergs.

\section{Expression for the Energy of Spinning Black Hole}

In 2005, Ram et al. concluded that the black hole is a Bose-Einstein ensemble of quanta of mass equals to twice the Planck mass, confined in a sphere of radius twice the black hole [6]. Quantum mechanically, however, there is a possibility that one of a particle production pair in a black hole is able to tunnel the gravitational barrier and escapes the black hole's horizon. Thus, a black hole is not really black; it can radiate or evaporate particles [7]. The space-time having a black hole in it, first, has a singularity, and second, has a horizon preventing an external observer from seeing it. The singularity in GR is radically different from field theory singularities because it is a property not of some field but

*Corresponding author: Dipo Mahto, Assistant Professor, Department of Physics, Marwari College, T.M.B.U. Bhagalpur-812007, India, Tel: 91141277 108; E-mail: dipomahto@hotmail.com

Received March 17, 2014; Accepted April 22, 2014; Published April 24, 2014

Citation: Mahto D, Nadeem MS, Prasad U, Vineeta K (2014) Energy of Spinning Black Holes in XRBs and AGN. Astrobiol Outreach 2:109. doi: 10.4172/2332 2519.1000109

Copyright: (C) 2014 Mahto D, et al. This is an open-access article distributed under the terms of the Creative Commons Attribution License, which permits unrestricted use, distribution, and reproduction in any medium, provided the original author and source are credited. 
of the space-time itself. The topology of space-time is changed when it acquires a black hole [8].

The black hole possesses an event horizon (a one-way membrane) that casually isolates the inside of the black hole from the rest of the universe. The radius of the event horizon of spinning black holes given by the Schwarzschild radius can be obtained as eq ${ }^{\mathrm{n}}$ (1) [9]. Event horizons are mathematically simple consequences of Einstein's general theory of relativity that were first pointed out by the German astronomer Karl Schwarzschild in a letter he wrote to Einstein in late 1915, less than a month after the publication of the theory. Quantum theory dictates that the event horizon must actually be transformed into a highly energetic region, or 'firewall', that would burn the astronaut to a crisp [10]. Now Hawking is suggesting a resolution to the paradox: Black holes do not possess event horizons after all, so they do not destroy information. Hawking says: "The absence of event horizons means that there are no black holes, in the sense of regimes from which light can't escape." In place of the event horizon, Hawking proposes that black holes possess "apparent horizons" that only temporarily entrap matter and energy that can eventually reemerge as radiation. This outgoing radiation possesses all the original information about what fell into the black hole, although in radically different form. Since the outgoing information is scrambled, Hawking writes, there's no practical way to reconstruct anything that fell in based on what comes out [11]. Hawking's new suggestion is that the apparent horizon is the real boundary [10], but the apparent horizon instead of the event horizon is a matter of discussion, so we consider the radius of event horizon of black holes.

$$
R_{s}^{\prime}=\frac{G M}{c^{2}}
$$

Hence the equation (1) can be transformed into the following equation.

$$
R_{s}^{\prime}=1475\left(M / M_{\varepsilon}\right) m
$$

The above equation can be transformed into the energy of black holes as [5].

$$
E_{B H s}=K_{B H s} R_{s}^{\prime}
$$

Where $E_{B H s}=M c^{2}=$ energy of the spinning black hole

\begin{tabular}{|c|c|c|c|c|}
\hline S. No & $\begin{array}{l}\text { Mass of } \\
B H_{s} \text { (M) }\end{array}$ & $\begin{array}{c}R_{s}^{\prime}=1475 \frac{M}{M_{\varepsilon}} \\
\text { (in metre) }\end{array}$ & $\begin{array}{l}\text { Black hole constant } \\
\left(\mathrm{K}_{\mathrm{BHs}}\right) \text { in } \mathrm{J} / \mathrm{m}\end{array}$ & $\begin{array}{c}\text { Energy of black } \\
\text { holes }\left(E_{\mathrm{BHs}}\right) \text { in ergs }\end{array}$ \\
\hline 1 & $5 \mathrm{M}_{0}$ & 7375 & $1.214 \times 10^{44}$ & $0.895 \times 10^{55}$ \\
\hline 2 & $6 \mathrm{M}_{0}$ & 8850 & $1.214 \times 10^{44}$ & $1.074 \times 10^{55}$ \\
\hline 3 & $7 \mathrm{M}_{0}$ & 10325 & $1.214 \times 10^{44}$ & $1.253 \times 10^{55}$ \\
\hline 4 & $8 \mathrm{M}_{0}$ & 11800 & $1.214 \times 10^{44}$ & $1.432 \times 10^{55}$ \\
\hline 5 & $9 \mathrm{M}_{0}$ & 13275 & $1.214 \times 10^{44}$ & $1.611 \times 10^{55}$ \\
\hline 6 & $10 \mathrm{M}_{0}$ & 14750 & $1.214 \times 10^{44}$ & $1.790 \times 10^{55}$ \\
\hline 7 & $11 \mathrm{M}_{0}$ & 16225 & $1.214 \times 10^{44}$ & $1.970 \times 10^{55}$ \\
\hline 8 & $12 M_{0}$ & 17700 & $1.214 \times 10^{44}$ & $2.149 \times 10^{55}$ \\
\hline 9 & $13 \mathrm{M}_{0}$ & 19175 & $1.214 \times 10^{44}$ & $2.328 \times 10^{55}$ \\
\hline 10 & $14 \mathrm{M}_{0}$ & 20650 & $1.214 \times 10^{44}$ & $2.507 \times 10^{55}$ \\
\hline 11 & $15 \mathrm{M}_{0}$ & 22125 & $1.214 \times 10^{44}$ & $2.686 \times 10^{55}$ \\
\hline 12 & $16 \mathrm{M}_{0}$ & 23600 & $1.214 \times 10^{44}$ & $2.865 \times 10^{55}$ \\
\hline 13 & $17 \mathrm{M}_{0}$ & 25075 & $1.214 \times 10^{44}$ & $3.044 \times 10^{55}$ \\
\hline 14 & $18 \mathrm{M}_{0}$ & 26550 & $1.214 \times 10^{44}$ & $3.223 \times 10^{55}$ \\
\hline 15 & $19 \mathrm{M}_{0}$ & 28025 & $1.214 \times 10^{44}$ & $3.402 \times 10^{55}$ \\
\hline 16 & $20 \mathrm{M}_{0}$ & 29500 & $1.214 \times 10^{44}$ & $3.581 \times 10^{55}$ \\
\hline
\end{tabular}

$$
\text { and } K_{B H s}=\frac{M_{\varepsilon} c^{2}}{1475}
$$

Table 1: Energy of spinning black holes in $\times$ RBs.

$$
\text { or } K_{B H s}=1.214 \times 10^{44} \mathrm{~J} / \mathrm{m}
$$

The term $K_{B H s}$ is designated as black hole constant for the spinning black hole and may be defined with the help of $\mathrm{eq}^{\mathrm{n}}$ (3). For this putting $R_{s}^{\prime}=1$ in the equation (3) and we have,

$$
E_{B H s}=K_{B H s}
$$

Hence Black hole constant of the spinning black hole may be defined as the energy of the spinning black hole for unit radius of the event horizon.

This constant has the vital role of calculating the energy of different spinning black holes for the given radius of the event horizon. This constant may be also used to calculate the change in energy, internal energy, entropy, enthalpy and other thermodynamical parameters of black holes.

\section{Data in the Support of the Mass and Energy of the Black Hole}

There are two categories of black holes classified on the basis of their masses clearly very distinct from each other, with very different masses $\mathrm{M} \sim 520 \mathrm{M} \odot$ for stellar - mass black holes in X-ray binaries (XRBs) and $\mathrm{M} \sim 10^{6}-10^{9.5} \mathrm{M}_{0}$ for super massive black holes in active galactic nuclei (AGN) $[9,12,13]$. Masses in the range $10^{6} \mathrm{M}_{0}$ to $3 \times 10^{9.5} \mathrm{M}_{0}$ have been estimated by this means in about 20 galaxies [14] and other data in the support of mass of black holes in AGN can be seen in the research paper [14,15] and for energy of black hole in the research paper [16,17]. On the basis of the data available for the mass of black holes, we have calculated the energy of spinning black holes in XRBs and AGN for the given radius of event horizon listed in Tables 1 and 2 respectively.

\section{Results and Discussion}

In the present paper, we have calculated the energy of different spinning black holes existing in XRBs and AGN on the basis of model( $E_{B H s}=K_{B H s} R_{s}^{\prime}$ ) The calculation shows that the total energy for the rest masses (M) lying between $5 \mathrm{M}_{0}$ to $20 \mathrm{M}_{0}$ in XRBs is few $\times 10^{55}$ ergs and for the rest masses $(\mathrm{M})$ between $10^{6} \mathrm{M}_{\circ}$ to $10^{9.5} \mathrm{M}_{\odot}$ of super massive black holes in AGN is few $\times 10^{60}-10^{64}$ ergs and agrees with the result of other research work done previously by Pacini and Salvati and by Krishan and justifies the validity of this model. The graphs have been plotted between: (i) the radius of event horizon $\left(R_{s}^{\prime}\right)$ of different spinning black holes and their corresponding energy in XRBs (Figure 1) (ii) the radius of event horizon $\left(R_{s}^{\prime}\right)$ of different spinning black holes and their corresponding energy in AGN (Figure 2).

Figures 1 and 2 obtained for XRBs and AGN are in a straight line showing the uniform variation between the radius of event horizon and their corresponding energy of spinning black holes. The straight line also shows that there is a linear relation between the radius of event horizon and energy of spinning black holes and justifies the validity of this model.

When the result of the present work is compared with the work in case of non-spinning black holes done by Mahto et al. (2013), we observe that the energy of spinning black holes for the same mass is the exactly equal which shows that the energy of black holes depends only on mass, not on the spinning.

The two models for the energy of non-spinning and spinning black holes as proposed by Kanak et al.(2010) and Mahto et al. (2011) respectively also confirms the validity of Einstein's mass-energy equivalence relation. 
Citation: Mahto D, Nadeem MS, Prasad U, Vineeta K (2014) Energy of Spinning Black Holes in XRBs and AGN. Astrobiol Outreach 2:109. doi: 10.4172/2332-2519.1000109

Page 3 of 4

\begin{tabular}{|c|c|c|c|c|c|c|}
\hline S. No. & Mass of $B H_{s}(\mathbf{M})$ & $R_{s}^{\prime}=1475 \frac{M}{M_{\varepsilon}}$ (in metre) & $\begin{array}{c}\log \left(R_{s}^{\prime}\right) \\
\text { (in metre) }\end{array}$ & $\mathrm{K}_{\mathrm{BHs}}($ in $\mathrm{J} / \mathrm{m})$ & $\begin{array}{l}\text { Energy of spinning black holes } \\
\qquad\left(E_{\mathrm{BHS}}\right) \text { in ergs }\end{array}$ & $\log \left(E_{B H S}\right)$ \\
\hline 1 & $1 \times 10^{6} \mathrm{M}_{0}$ & $1.475 \times 10^{9}$ & 9.1687 & $1.214 \times 10^{44}$ & $1.790 \times 10^{60}$ & 60.2529 \\
\hline 2 & $2 \times 10^{6} \mathrm{M}_{0}$ & $2.950 \times 10^{9}$ & 9.4698 & $1.214 \times 10^{44}$ & $3.612 \times 10^{60}$ & 60.5565 \\
\hline 3 & $3 \times 10^{6} \mathrm{M}_{0}$ & $4.425 \times 10^{9}$ & 9.6459 & $1.214 \times 10^{44}$ & $5.372 \times 10^{60}$ & 60.7302 \\
\hline 4 & $4 \times 10^{6} \mathrm{M}_{0}$ & $5.900 \times 10^{9}$ & 9.7708 & $1.214 \times 10^{44}$ & $7.163 \times 10^{60}$ & 60.8551 \\
\hline 5 & $5 \times 10^{6} \mathrm{M}_{0}$ & $7.375 \times 10^{9}$ & 9.8677 & $1.214 \times 10^{44}$ & $8.954 \times 10^{60}$ & 60.9520 \\
\hline 6 & $6 \times 10^{6} \mathrm{M}_{0}$ & $8.850 \times 10^{9}$ & 9.9469 & $1.214 \times 10^{44}$ & $1.074 \times 10^{61}$ & 61.0310 \\
\hline 7 & $7 \times 10^{6} \mathrm{M}_{0}$ & $1.032 \times 10^{10}$ & 10.0136 & $1.214 \times 10^{44}$ & $1.253 \times 10^{61}$ & 61.0979 \\
\hline 8 & $8 \times 10^{6} \mathrm{M}_{0}$ & $1.180 \times 10^{10}$ & 10.0718 & $1.214 \times 10^{44}$ & $1.432 \times 10^{61}$ & 61.1559 \\
\hline 9 & $9 \times 10^{6} \mathrm{M}_{0}$ & $1.327 \times 10^{10}$ & 10.1228 & $1.214 \times 10^{44}$ & $1.611 \times 10^{61}$ & 61.2071 \\
\hline 10 & $1 \times 10^{7} M_{0}$ & $1.475 \times 10^{10}$ & 10.1687 & $1.214 \times 10^{44}$ & $1.790 \times 10^{61}$ & 61.2529 \\
\hline 11 & $2 \times 10^{7} \mathrm{M}_{0}$ & $2.950 \times 10^{10}$ & 10.4698 & $1.214 \times 10^{44}$ & $3.612 \times 10^{61}$ & 61.5565 \\
\hline 12 & $3 \times 10^{7} \mathrm{M}_{0}$ & $4.425 \times 10^{10}$ & 10.6459 & $1.214 \times 10^{44}$ & $5.372 \times 10^{61}$ & 61.7302 \\
\hline 13 & $4 \times 10^{7} \mathrm{M}_{0}$ & $5.900 \times 10^{10}$ & 10.7708 & $1.214 \times 10^{44}$ & $7.163 \times 10^{61}$ & 61.8551 \\
\hline 14 & $5 \times 10^{7} \mathrm{M}_{0}$ & $7.375 \times 10^{10}$ & 10.8677 & $1.214 \times 10^{44}$ & $8.954 \times 10^{61}$ & 61.9520 \\
\hline 15 & $6 \times 10^{7} \mathrm{M}_{0}$ & $8.850 \times 10^{10}$ & 10.9469 & $1.214 \times 10^{44}$ & $1.074 \times 10^{62}$ & 62.0310 \\
\hline 16 & $7 \times 10^{7} \mathrm{M}_{0}$ & $1.032 \times 10^{11}$ & 11.0136 & $1.214 \times 10^{44}$ & $1.253 \times 10^{62}$ & 62.0979 \\
\hline 17 & $8 \times 10^{7} M_{0}$ & $1.180 \times 10^{11}$ & 11.0718 & $1.214 \times 10^{44}$ & $1.432 \times 10^{62}$ & 62.1559 \\
\hline 18 & $9 \times 10^{7} \mathrm{M}_{0}$ & $1.327 \times 10^{11}$ & 11.1228 & $1.214 \times 10^{44}$ & $1.611 \times 10^{62}$ & 62.2071 \\
\hline 19 & $1 \times 10^{8} \mathrm{M}_{0}$ & $1.475 \times 10^{11}$ & 11.1687 & $1.214 \times 10^{44}$ & $1.790 \times 10^{62}$ & 62.2529 \\
\hline 20 & $2 \times 10^{8} \mathrm{M}_{0}$ & $2.950 \times 10^{11}$ & 11.4698 & $1.214 \times 10^{44}$ & $3.162 \times 10^{62}$ & 62.5565 \\
\hline 21 & $3 \times 10^{8} \mathrm{M}_{0}$ & $4.425 \times 10^{11}$ & 11.6459 & $1.214 \times 10^{44}$ & $5.372 \times 10^{62}$ & 62.7302 \\
\hline 22 & $4 \times 10^{8} \mathrm{M}_{0}$ & $5.900 \times 10^{11}$ & 11.7708 & $1.214 \times 10^{44}$ & $7.163 \times 10^{62}$ & 62.8551 \\
\hline 23 & $5 \times 10^{8} \mathrm{M}_{0}$ & $7.375 \times 10^{11}$ & 11.8677 & $1.214 \times 10^{44}$ & $8.954 \times 10^{62}$ & 62.9520 \\
\hline 24 & $6 \times 10^{8} \mathrm{M}_{0}$ & $8.850 \times 10^{11}$ & 11.9469 & $1.214 \times 10^{44}$ & $1.074 \times 10^{63}$ & 63.0310 \\
\hline 25 & $7 \times 10^{8} \mathrm{M}_{0}$ & $1.032 \times 10^{12}$ & 12.0136 & $1.214 \times 10^{44}$ & $1.253 \times 10^{63}$ & 63.0979 \\
\hline 26 & $8 \times 10^{8} \mathrm{M}_{0}$ & $1.180 \times 10^{12}$ & 12.0718 & $1.214 \times 10^{44}$ & $1.432 \times 10^{63}$ & 63.1559 \\
\hline 27 & $9 \times 10^{8} \mathrm{M}_{0}$ & $1.327 \times 10^{12}$ & 12.1228 & $1.214 \times 10^{44}$ & $1.611 \times 10^{63}$ & 63.2071 \\
\hline 28 & $1 \times 10^{9} \mathrm{M}_{0}$ & $1.475 \times 10^{12}$ & 12.1687 & $1.214 \times 10^{44}$ & $1.790 \times 10^{63}$ & 63.2529 \\
\hline 29 & $2 \times 10^{9} \mathrm{M}_{0}$ & $2.950 \times 10^{12}$ & 12.4698 & $1.214 \times 10^{44}$ & $3.612 \times 10^{63}$ & 63.5565 \\
\hline 30 & $3 \times 10^{9} \mathrm{M}_{0}$ & $4.425 \times 10^{12}$ & 12.6459 & $1.214 \times 10^{44}$ & $5.372 \times 10^{63}$ & 63.7302 \\
\hline 31 & $4 \times 10^{9} \mathrm{M}_{0}$ & $5.900 \times 10^{12}$ & 12.7708 & $1.214 \times 10^{44}$ & $7.163 \times 10^{63}$ & 63.8551 \\
\hline 32 & $5 \times 10^{9} \mathrm{M}_{0}$ & $7.375 \times 10^{12}$ & 12.8677 & $1.214 \times 10^{44}$ & $8.954 \times 10^{63}$ & 63.9520 \\
\hline
\end{tabular}

Table 2: Energy of spinning black holes in AGN.

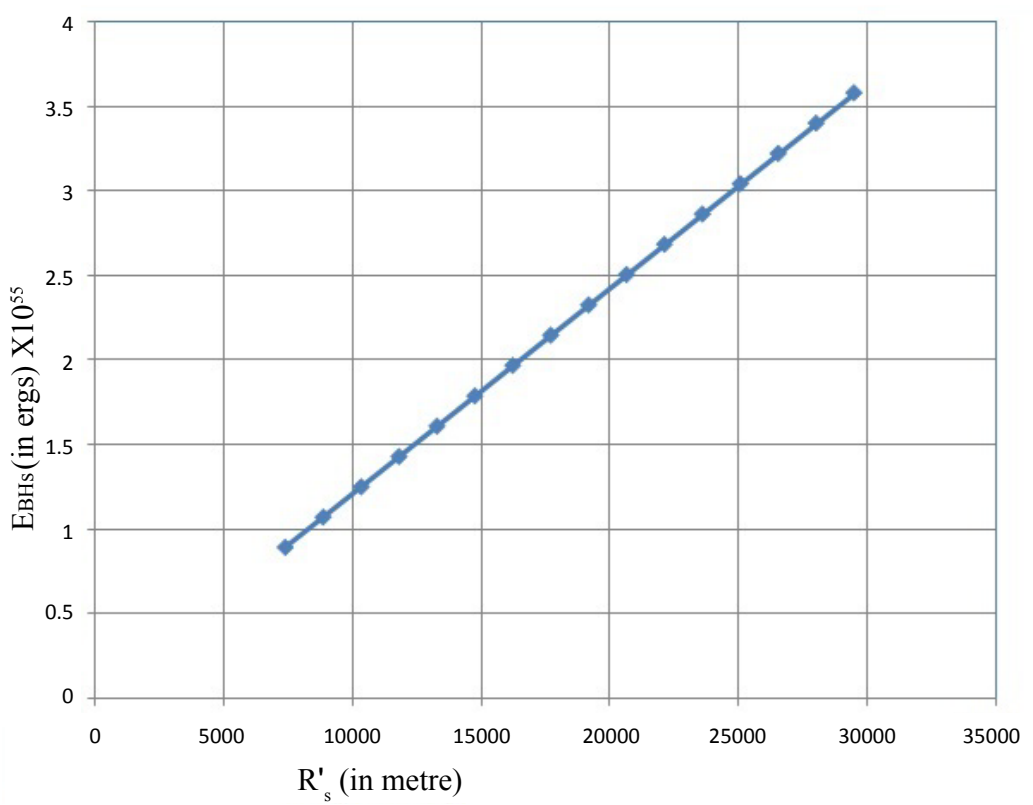

Figure 1: The graph plotted bet the radius of event horizon and corresponding energy of spinning black hole in XRBs (Table1) 
Citation: Mahto D, Nadeem MS, Prasad U, Vineeta K (2014) Energy of Spinning Black Holes in XRBs and AGN. Astrobiol Outreach 2:109. doi: 10.4172/2332-2519.1000109

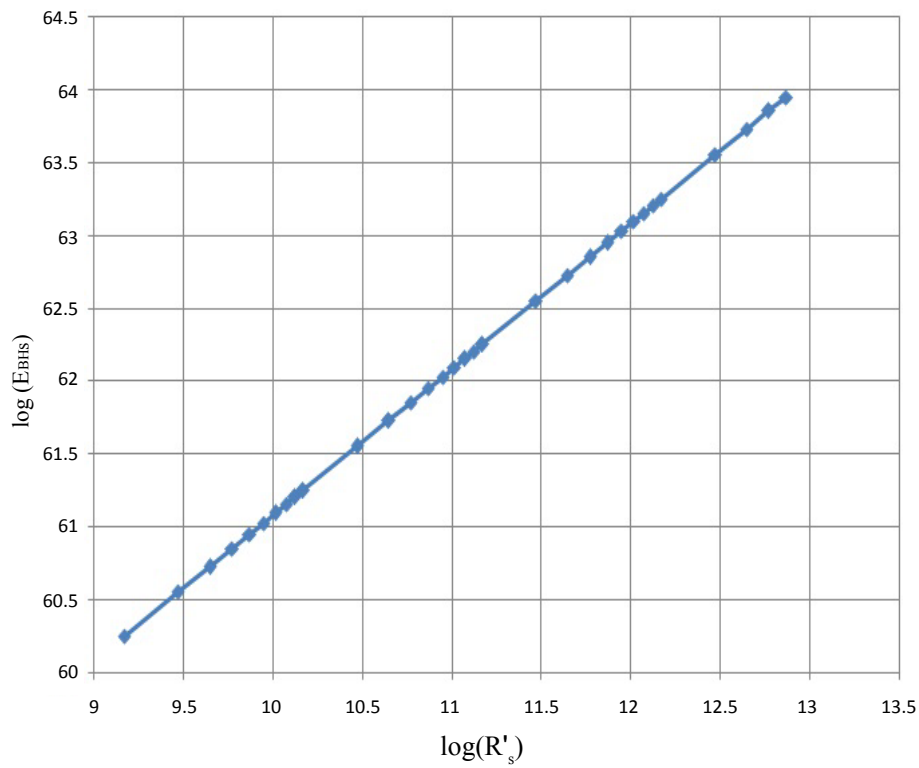

Figure 2: The graph plotted bet the radius of event horizon and corresponding energy of spinning black hole in agn (Table 2).

The discussion of present work requires the recent comments of Dr. Hawking on the non-existence of event horizon of black holes.

\section{Conclusion}

In the light of the present research work, we can draw the following conclusions:

1. There is a uniform variation between the radius of event horizon and energy of spinning black holes.

2. The straight line also shows that there is a definite relation between the radius of event horizon and the energy of spinning black holes and gives the validity of model $\left(E_{B H s}=K_{B H s} R_{s}^{\prime}\right)$ as proposed by Mahto et al. (2011).

3. The energy calculated on the basis of model $E_{B H s}=K_{B H s} R_{s}^{\prime}$ agrees with the result of other research work done earlier by Pacini andSalvati (1982) and Krishan (1985), which also confirms the validity of model $\left(E_{B H s}=K_{B H s} R_{s}^{\prime}\right)$ as proposed by Mahto et al.(2011).

4. The larger the radius of event horizon, the greater is the energy of black holes and vice versa.

5. The energy of black holes depends only on mass, not on the spinning.

\section{References}

1. Bardeen JM, Carter B, Hawking SW (1973) The four laws of black hole mechanics. Commun Math Phys 31: 161-170

2. Hawking SW (1975) Particle creation by black holes, Commun. Math. Phys 43: $199-220$

3. Mahto D, Kumari K, Sah RK, Singh KM (2011) Study of non-spinning black holes with reference to the change in Energy and Entropy. Astrophys Space Sci 4:1-6.

4. Energy from a black hole: NASA Science.

5. Mahto D, Kumari K, Sah RK, Ved Prakash (2011) Study of Schwarzschild radius with reference to the spinning black holes. Bulletin of pure and Applied Sciences 30: 157-162.
6. Ram B, Ram A, Ram N (2005) The quantum black hole.

7. Triyanta, Bowaire AN (2013) Hawking Temperature of the Reissner-NordstromVaidya Black Hole. J. Math. Fund. Sci. 45: 114-123.

8. Soloviev VO (2005) Black hole Statistical Physics: Entropy. Institute for High Energy Physics, Protvino, Russia.

9. Narayan R (2005) Black Holes In Astrophysics. New Journal Physics 7: 1-31.

10. Stephen Hawking (2014) No Black Holes Exist.

11. Editor's note (Feb15,2014) "The Truth About Black Holes" in the March issue of National Geographic magazine.

12. Mahto D, Nadeem MS, Prasad U, Kumar A, Singh KM (2013) Change in entropy of non-spinning black holes w.r.t. the radius of event horizon in AGN. Journal of Modern Physics 4: 321-326.

13. Mahto D, Mehta RN, Pant N, Sah RK (2013) Change in Energy of Non-spinning Black Holes w.r.t. the Change in Mass. International Journal of Astronomy and Astrophysics 3: 49-56

14. Mahto D, Rama NM, Kamla P, Krishna MS, Raj KS (2013) Energy of the nonspinning black holes in XRBs and AGN. Scientific Journal of Physical Science 3: $1-5$.

15. RFerrarese L, Ford H (2005) Super massive Black Holes in Galactic Nuclei: Past Present \& Future Research. Space Science Reviews 116:523-624.

16. Pacini V, Salvati M (1982) The nature of the Energy Source in Radio Galaxies and Active Galactic Nuclei. International Astronomical Union 97:247-253.

17. Krishan V (1985) Acceleration and Radiation processes around Active Galactic Nuclei. Astrophysics and Space Science 115:119-126 